\title{
Shape of Nascent and Completed Poles of Bacillus subtilis
}

\author{
By I. D. J. BURDETT ${ }^{1 *}$ AND A. L. $\mathrm{KOCH}^{2}$ \\ ${ }^{1}$ National Institute for Medical Research, The Ridgeway, Mill Hill, London NW7 1AA, UK \\ ${ }^{2}$ Department of Biology, Indiana University, Bloomington, Indiana 47405, USA
}

(Received 17 October 1983; revised 30 January 1984)

\begin{abstract}
The zonal dome model has previously been shown to fit the shape of the poles of the Grampositive Streptococcus faecium quite accurately, but measurements of the angle that the pole of Bacillus subtilis makes with the cylinder portion of the cell show that the poles of this organism do not fit this model. Furthermore, the diameters of curvatures in different parts of the completed and nascent pole are contrary to the predictions of the zonal dome model and several other proposed models. The results suggest that the poles of Gram-positive rods are generated by a mechanism designated the 'split-and-stretch' model. The model assumes that no additional murein is inserted as the septal wall is split and externalized.
\end{abstract}

\section{INTRODUCTION}

Wall growth of Streptococcus faecium takes place by inward formation of the septum and by addition of new material at the site of septum splitting (Higgins \& Shockman, 1976). We have proposed (Koch et al., 1981a) that in the narrow zone at the site of septum splitting, pole formation is controlled by the same laws as those that govern the enlargement of a soap film. This assumes that the external wall is plastic only in this narrow zone where new external wall is incorporated during the splitting process. On the further assumptions that the intracellular hydrostatic pressure, $P$, and surface tension, $T$, at the growth zone are constant and that the septum at the splitting region is twice as wide as the externalized wall, a formula was derived which predicted the shape of $S$. faecium.

By studying median sections of Bacillus subtilis, we report here that the zonal dome model fits the pole shape of this Gram-positive rod very poorly. The data presented here led to a new model, designated the 'split-and-stretch' model, which assumes that no new murein is added to the septum as it splits, but that the septal wall is capable of an approximately twofold increase in area when stressed. Thus the 'split-and-stretch' model assumes that the conformation of bonds assembled in the septal wall is less extended than the maximally extended conformation. However, eventually the bonds in the yet unsplit septal wall do become extended sometime after being stressed. This allows the initial flat septum to achieve a series of convex shapes.

\section{ANALYSIS OF ELECTRON MICROGRAPHS}

In electron micrographs of B. subtilis, Burdett \& Higgins (1978) observed 'raised tears', perhaps analogous to the wall bands of streptococci (Higgins \& Shockman, 1976). These can be used to distinguish the boundary between nascent or completed polar wall and the cylindrical wall. During pole enlargement, the wall bands become less conspicuous, perhaps due to loss of wall material by turnover or sloughing. In completed, fully separated poles, the bands are not usually visible and we have delimited where they should be by placing a straight edge on the cylindrical portion of median, axial sections of the wall and marking the presumptive point where the pole commences. In those rare cells where wall bands have been observed at the poles 


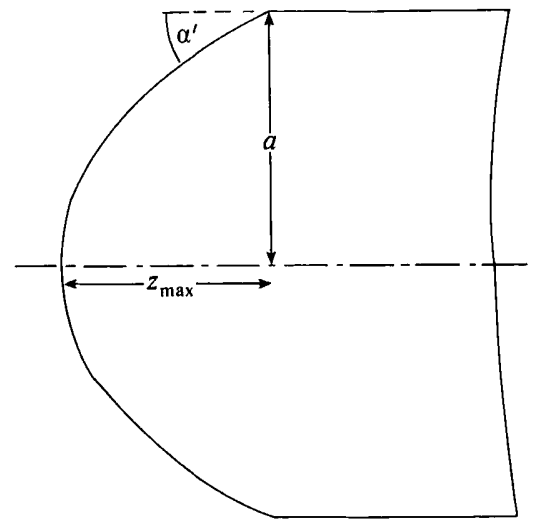

Fig. 1. The profile of the pole of Bacillus subtilis predicted by the zonal dome model. The pole or cap and a portion of the cylindrical side walls are shown. The curve was calculated to fit the average measurements of 93 non-septating cells. The mean radius of the pole, $a$, was $0.2868 \mu \mathrm{m}$; the mean axial height of the pole, $z_{\max }, 0.2363 \mu \mathrm{m}$. The angle, $\alpha^{\prime}$, that the pole makes with the cylinder wall, predicted by the zonal dome model, of $25 \cdot 7^{\circ}$, is indicated.

distal to the developing cross-wall, the bands appeared to lie at the same location as that determined by using a straight edge.

The zonal dome model predicts an abrupt change in slope of the cell profile at the wall band. The angle between the tangent to the pole at the intersection with the cylinder is designated $\alpha$ or $\alpha^{\prime}$ (see Fig. 1). In the measurements presented here, the experimental angle, $\alpha$, was measured by aligning a protractor with the first $1-2 \mathrm{~mm}$ of the pole. The zonal dome model also predicts that the mean value of $\alpha$ for old poles should equal the mean value $\alpha$ of nascent poles; consequently, $\alpha$ for nascent poles was also measured.

Measurements were made of the width and the diameter of curvature in different portions of the cylinder and completed and nascent poles. The curvature of the median sections was estimated by superimposing plastic metric circular curves used for drafting (Linex 1116, Denmark; Radien-Tangenten 842 687, Rotring, FRG) (see Koch, 1983). The latter was particularly useful because a diameter of curvature could be selected that was the best visual fit, subject to the constraint that the circular curve is tangent to the cylindrical part of the cell. The best fit could also be visually restricted to a specified arc of the curve. In this way, the portion of the developing pole and the completed pole making the lateral $45^{\circ}$ of arc tangent to the cylinder of a cell was measured. We also measured the $90^{\circ}$ of arc at the end of the pole. These measures were all expressed as diameters of curvature for each bacterium. The electron micrographs were also traced on a tablet connected to a computer as previously described (Burdett, 1980 b). Data were retrieved and manipulated also as previously described. The resultant data base in the computer contained enough information so that diameter of curvature of the entire nascent pole (from wall band to notch) for the nearly divided cells could also be calculated.

The sections of $B$. subtilis used in the present study were prepared for electron microscopy by methods described by Burdett \& Higgins (1978) and Burdett $(1980 \mathrm{~b})$. The regimes used for fixation, dehydration, and embedding undoubtedly lead to cell shrinkage. Arguments for believing that valid, albeit relative, dimensions can be obtained from such preparations have been discussed extensively by Higgins (1976) and Burdett \& Higgins (1978); these papers also discuss the accuracy of the methods of measurement.

The pole of the rod, B. subtilis, is blunter than the coccus, $S$. faecium, and poles of the former [such as the one shown in Fig. 6B of Burdett \& Higgins (1978) or Fig. 3 of this paper], if fitted to the zonal dome shape, require values of $P / T$ in the range of $4-5$, compared with a value of 1.54 for $S$. faecium. 


\section{TEST OF THE ZONAL DOME MODEL FOR COMPLETED POLES}

\section{Angle measurements}

For a zonal dome-shaped pole, the ratio of axial height, $z_{\max }$, to base radius, $a$ [see equation (5), Koch et al., 1981a] is given by:

$$
z_{\max } / a=-\ln \left[1-(P a / 2 T)^{2}\right] /(P a / 2 T)
$$

This equation can be solved for $\mathrm{Pa} / \mathrm{T}$ given $z_{\max } / a$ either by graphical means using Fig. 2 or by numerical techniques. We used an efficient search procedure which will be transmitted to those desiring the method.

From the mean value of $P a / T$, the slope, and hence the predicted angle, $\alpha^{\prime}$, or the slope, $S$, that the zonal dome makes with the cylindrical wall (at the wall band) can be calculated according to equation (3) of Koch et al. (1981a):

$$
\tan \left(\alpha^{\prime}\right)=S=P a / 4 T-T / P a
$$

Consequently, an observed value of $z_{\max } / a$ leads to a predicted value of the angle $\alpha^{\prime}$ (see Fig. 2), which can then be compared with the observed angle $\alpha$.

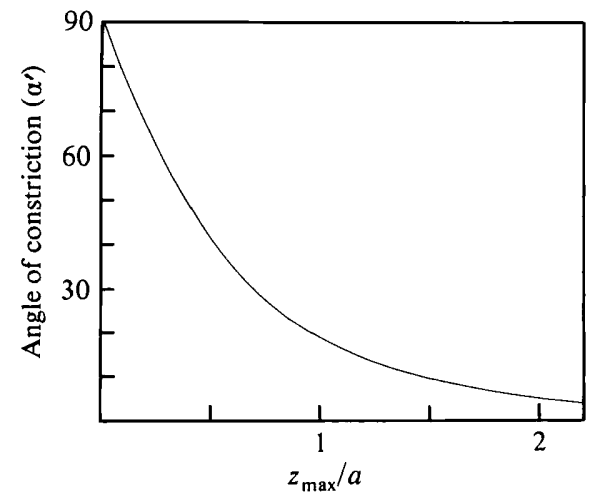

Fig. 2. Relationship of $\alpha^{\prime}$ with $z_{\max } / a$. The graph was computed from the relationships given in the text; the quantities are defined in Fig. 1.

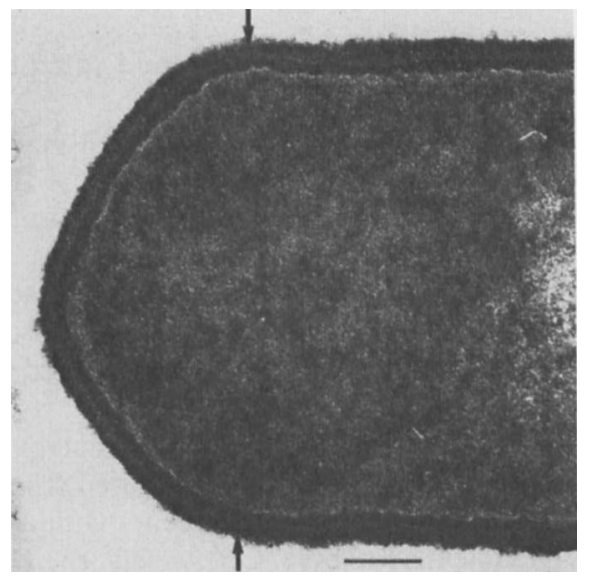

Fig. 3. Electron micrograph of a portion of a median, longitudinal section of $B$. subtilis strain $168 \mathrm{~S}$ taken from a culture of exponential growth in glucose minimal medium (doubling time $60 \mathrm{~min}$ ) and fixed with glutaraldehyde $/ \mathrm{OsO}_{4}$, as described by Burdett \& Higgins (1978). Arrows indicate presumed junction of pole with the cylindrical portion of the cell. Bar marker, $0.1 \mu \mathrm{m}$. This section is presumed to be a median one because it had a tribanded appearance of the wall over the entire profile of the wall. 
Table 1. Comporison of the mean observed and the predicted angle of the zonal dome model

\begin{abstract}
The two observed angles for each pole were averaged and used as a datum in statistical calculations of the mean observed angle, $\alpha$. The predicted mean angle, $\alpha^{\prime}$, was calculated for each pole from the observed $z_{\max }$, the axial height, and $2 a$, the width of the cell at the pole. The numbers of cells in each category are given in Table 2.
\end{abstract}

$\begin{array}{clc} & & \text { Mean } \pm \text { SE } \\ \text { Smallest cells } & \alpha & 12 \cdot 0^{\circ} \pm 0 \cdot 5^{\circ} \\ & \alpha^{\prime} & 27 \cdot 8^{\circ} \pm 0 \cdot 6^{\circ} \\ \text { Septum-initiating cells } & \alpha & 11 \cdot 7^{\circ} \pm 0 \cdot 4^{\circ} \\ & \alpha^{\prime} & 27.0^{\circ} \pm 0 \cdot 6^{\circ} \\ \text { Nearly divided cells } & & \\ \text { completed pole } & \alpha & 13 \cdot 0^{\circ} \pm 0 \cdot 5^{\circ} \\ \text { nascent pole } & \alpha^{\prime} & 27 \cdot 2^{\circ} \pm 0 \cdot 5^{\circ} \\ & \alpha & 17 \cdot 6^{\circ} \pm 0 \cdot 6^{\circ} \\ & \alpha^{\prime} & 27.2^{\circ} \pm 0 \cdot 5^{\circ}\end{array}$

We measured $z_{\max }, 2 a$, and the two $\alpha$ values for the 158 poles of 79 cells for which good longitudinal, median sections were available. These sections were chosen from tens of thousands of cell sections to include median sections of different short phases of pole formation. The statistics of the experimental measurements are presented in Table 1. The cells examined belonged to three subclasses: smallest cells, cells possessing the early beginnings of a septum, and nearly divided cells where the septum was almost completely split. The two angles between the junction of a pole with the cylindrical regions for each pole were averaged. This angie was compared with $\alpha^{\prime}$ calculated from the measured values of axial height $\left(z_{\max }\right)$ and the pole width $(2 a)$. The combined mean value for completed poles of the three subclasses was $12.7^{\circ}$ and that expected from the zonal dome model is $27 \cdot 3^{\circ}$. This lack of agreement of $\alpha$ and $\alpha^{\prime}$ represents strong evidence against the zonal dome model for $B$. subtilis.

\title{
Diameter of curvature measurements
}

For a given mathematical relationship, the diameter of curvature can be computed readily from the first and second derivative. For the zonal dome equation, the diameter of curvature, $D_{\mathrm{c}}$, can be shown to be:

$$
D_{\mathrm{c}}=(T / P)\left[\left(1-[\operatorname{Pr} / 2 T]^{2}\right) /\left(1+[\operatorname{Pr} / 2 T]^{2}\right)\right] /\left[1+4[\operatorname{Pr} / T]^{2} /\left(1-[\operatorname{Pr} / 2 T]^{2}\right)^{2}\right]^{3 / 2}
$$

For a hemispherical pole, the diameter of curvature should equal the width of the cylindrical pole; i.e. $D_{\mathrm{c}}=2 a$.

The experimentally measured values are summarized in Table 2 for the three subclasses of cells. In addition, the average measurements are shown in Fig. 4. This figure and Figs 5 and 6 show one-half of the average profile of the pole assuming axial symmetry. For the smallest cells, each cell was oriented so that the left side was the one that appeared to be more regular. A very few poles were so irregular that they were discarded before the statistics were computed. In Table 2, the data from the two halves of the cells were combined. Also shown in the table are pole curvatures calculated from the pole surface area for the completed poles and from the contour length of the split septum of the nascent pole.

For a given subclass, the width of the cell is quite constant for different positions of measurement. In all cases, the diameter of curvature measured from the end of the cylinder to $45^{\circ}$ is significantly smaller than the diameter of the adjacent cylinder. Thus the $D_{\mathrm{c}}$ near the sides is smaller than expected for a hemispherical pole. Conversely, the $D_{\mathrm{c}}$ measured over the central $90^{\circ}$ arc (i.e. from $-45^{\circ}$ to $+45^{\circ}$ ) is in all cases larger than the cylinder width. These two kinds of measurements concur that most of the curvature needed to complete the arc of the pole occurs near the edges of the pole. This effect is further heightened for the nascent pole; this can be seen in the published photographs of Burdett \& Higgins (1978) (see particularly their Figs 4A and 6). Initially, the splitting septum is much more sharply curved than is the part that is later split to 
Table 2. Linear dimensions of glucose-grown exponential phase cells of $B$. subtilis $168 S$

All dimensions expressed as diameters. All errors are standard errors. If the cells were formed as cylinders with hemispherical poles, all entries would be equal. A very few poles were so irregular that they were omitted from the statistics.

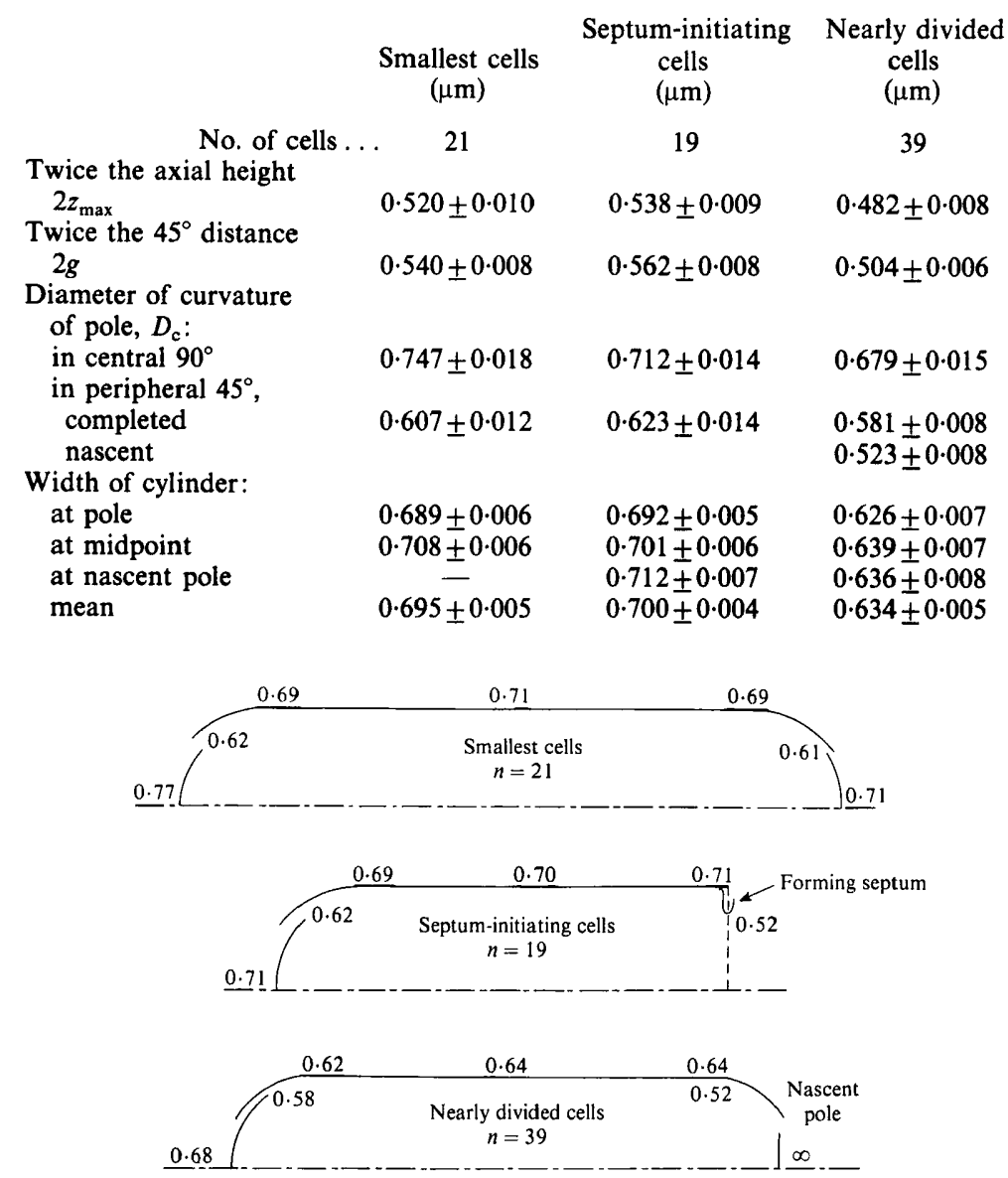

Fig. 4. Mean profile of the three classes of $B$. subtilis cells. The average dimensions are shown for the different classes of cells. For the smallest cells, the most regular pole was oriented on the left side. Circular arcs are shown covering the appropriate regions of the cell. Dimensions of the mean diameter of curvature measurements in $\mu \mathrm{m}$ are given.

separate the two daughter cells. The latter region of the pole is, in fact, nearly planar. To repeat: the pole of $B$. subtilis is blunter than a hemisphere, as if it had been flattened at the tip.

In Fig. 5 , the pole profiles of the average dimensions of completed poles and the nearly divided nascent pole are plotted to define the profile of one half of a pole. The reader must imagine the three-dimensional pole that would result if the curves shown in Figs 5-8 were rotated around the $x$ axis. Instead of depicting the diameters of curvature in Fig. 5 as circular arcs, these have been plotted as distances at an angle of $22.5^{\circ}$ from the axis for the measurement of the central $90^{\circ}$ of arc of the pole and at $67.5^{\circ}$ from the axis for the measurement of the peripheral $45^{\circ}$ of arc. These angles correspond to the mean angle for the measurements. In toto, these measures define the average pole shape quite precisely.

Ideally, we should like to be able to group together only completed poles of a specified age range. Methods have not been implemented that would allow us to estimate the age of the completed pole observed in the electron microscope, but we can use statistical techniques to unravel the history of the pole. Septum-initiating cells are in the earliest stage of the division 


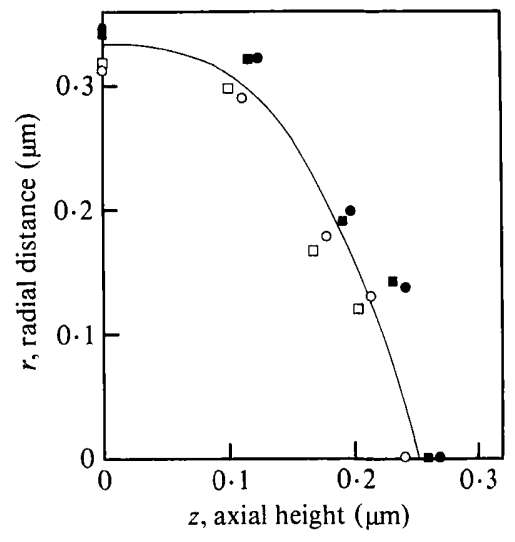

Fig. 5

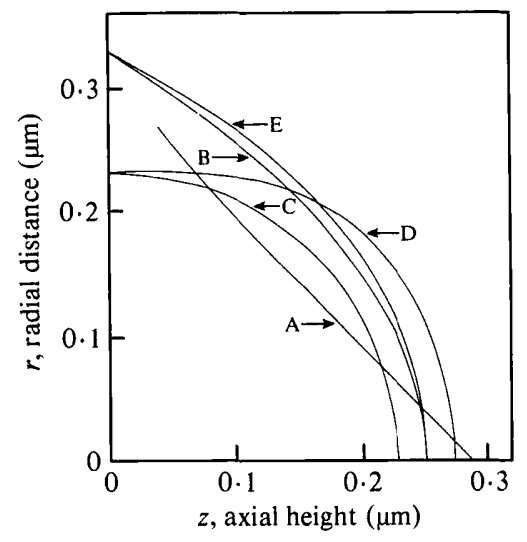

Fig. 6

Fig. 5. Experimentally measured pole profiles. The curves shown in this and the remaining figures of this paper should be thought of as meridian sections that on rotation around the $x$ axis would reconstruct a pole. The average dimensions in $\mu \mathrm{m}$ of the completed poles of cells in the three categories are shown. See text for explanation of basis of plotted curvature data at $22.5^{\circ}$ and $67 \cdot 5^{\circ}$ from the axis of the cell. $\bigcirc$, Smallest cells; $O$, septum-initiating cells; $\square$, nascent poles of nearly divided cells; completed poles of nearly-divided cells.

Fig. 6. Pole shapes for B. subtilis according to the literature. Curve A, polynomial fit of Barrett \& Burdett (1981) to measurement of 118 poles. Curve B, best parabola oriented with its axis parallel to the cell axis. The parameters for the fit were the mean measurements for completed poles of the 79 cells analysed in the present paper. Curve $C$, the least-square fit to an elliptical model. The parameters were those fitted to an electron micrograph chosen by Oldmixon (1974). Curve D, least-square fit to a Cassinian model of the same cell according to Oldmixon's calculated parameters. Curve E, best zonal dome fit to data for all completed poles in this study based on the average value of $a$ and $z_{\max }$.

cycle, but provide only measurements of the initial width, $2 a$. The nascent poles of the nearly divided cells are next youngest. One of the two poles of each of the smallest cells is very young; however, the other pole can have any age. Statistically, half of these ends are a little more than one generation old, and one-quarter are little more than two generations old, etc. [We tried to see if the poles might become either more or less regular with development and therefore oriented the cells in a consistent way with respect to this criterion, but on statistical analysis the two ends differed little. This in itself would suggest that the poles are invariant in shape and size after formation.] The ages of completed poles of the nearly divided cells are, on average, nearly a generation older than the age of completed poles of the smallest cells. In both Table 2 and Fig. 5, it is evident that the widths and curvatures do change during the cell cycle, but the proportions stay quite constant. For example, $D_{\mathrm{c}}$ for the central $90^{\circ}$ of arc is 88,89 , and $92 \%$ of the mean widths of cylinders for the three populations. The agreement of the relative dimensions for completed poles in the three categories of cells seems to indicate that there is little change in shape occurring after a pole is completed.

\section{COMPARISON OF NASCENT AND COMPLETED POLES IN THE SAME NEARLY DIVIDED CELL}

Although, after completion, the pole shape seems fairly constant, it clearly changes significantly during its morphogenesis; the nascent poles of the nearly divided cells are quite different in angle and $D_{\mathrm{c}}$ measurements when compared with the completed poles of the same cell. Early in the development of a pole, there is a very clear discontinuity in slope at the junction of cylinder and developing pole. But very quickly this discontinuity becomes harder to discern, and it is for this reason that we supplemented angular measurements with measurement of the diameters of curvature measured with the restriction that the chosen circle be tangent to the cylinder portion of the cell and only $45^{\circ}$ of arc be considered. 
For each of the four poles of the 39 nearly divided cells in the sample, the two angles that the nascent poles make with the cylindrical region and the four angles that the completed poles make with the cylinder were measured. For each cell, the values for upper and lower parts of the pole were averaged, and the statistical analysis shown in the legend of Table 1 was performed. For all cells, the angle was more acute for the nascent than for the completed pole. The mean and its standard error was $-17 \cdot 6^{\circ} \pm 0 \cdot 6^{\circ}$ for the nascent poles and $-13 \cdot 6^{\circ} \pm 0 \cdot 5^{\circ}$ for the completed poles, respectively. The same conclusion can be drawn from the curvature measurements given in Table 2: the $D_{\mathrm{c}}$ measured in the $45^{\circ}$ of arc near the cylinder for the nascent poles of nearly divided cells is $0.523 \pm 0.008 \mu \mathrm{m}$, while that for the completed poles of the same sample is 0.581 $\pm 0.008 \mu \mathrm{m}$. Clearly, both angular and curvature measurements show differences that are highly significant. Consequently, the pole changes shape significantly, either during the remainder of the separation process or shortly after cell separation. This result rules out the zonal dome model in a different way than in previous sections. In fact, it rules out the entire class of models that assume that the pole shape is determined as the septum splits and that the wall in all other regions, once formed, is rigid.

\section{CONCLUSIONS}

Theoretical reasons for expecting that the poles of $B$. subtilis would be rigid after formation have been published elsewhere (Koch et al., 1981 b, 1982; Koch, 1983). The present experimental evidence demonstrating that the nascent pole changes shape during formation is convincing evidence against the zonal dome model for $B$. subtilis, but since the evidence also indicates that poles only change shape to a small degree after formation, the current work is probably consistent with the assumption that the poles can serve as a rigid framework on which diffuse inside-to-outside growth of the side walls can take place. Before returning to this issue, we show below how the present measurements of completed poles are inconsistent with all shapes proposed in the literature.

\section{Previous attempts to fit the pole shape of $B$. subtilis to various growth models}

Figure 6 shows fits, to data of previous investigators or to those data presented here according to five different models proposed in the literature. The first fit is to the data of Burdett \& Higgins (1978) as given in Barrett \& Burdett (1981). The latter authors made polynomial fits to the dimensions of 118 cells in various stages of division and found:

$$
\begin{gathered}
D_{\min }=0.538-0.197 \mathrm{P}_{a}-2.54 \mathrm{P}_{a}{ }^{2} \\
z=0.039+0.033 \mathrm{P}_{a}+1.3 \mathrm{P}_{a}{ }^{2}
\end{gathered}
$$

where $D_{\min }$ is the diameter at the bases of the cross-wall, $z$ is the axial height, and $\mathbf{P}_{a}$ is the pole area estimated by mathematical reconstruction on the assumption of cylindrical symmetry. These equations are presented again here because there were typographical errors in the original paper; the calculated pole is shown by curve $\mathrm{A}$ in Fig. 6 . It was calculated by choosing $\mathrm{P}_{a}$ values arbitrarily and calculating pairs of $z, r\left(=D_{\min } / 2\right)$ from equations (4) and (5). We conclude that the fit is very poor not only because the shape is not realistic, but because it predicts a non-zero value of $z$ when $P_{a}$ is zero and because it predicts a pointed tip to the pole.

Barrett \& Burdett (1981) also tested several simple geometric shapes. Primarily using data expressing the quantity $C$, which Burdett \& Higgins (1978) had defined as the difference along the nascent pole from notch to wall band minus the chord distance divided by the chord distance, they found that a parabola gave the best fit of all the conic sections to their experimental curvature data. Although they found that the parabola fitted the $C$ data best, the estimates of the parameters of the best fitting parabola were not given. Curve B in Fig. 6 shows the parabola for the parameters $z_{\max }=0.2528 \mu \mathrm{m}$ and $a=0.3294 \mu \mathrm{m}$ for the case in which the axis of the parabola is colinear with the axis of the cell. These values are the averages for the completed pole of the 79 cells studied here. The fit is not bad, but severe additional problems arise for the parabolic fit as proposed by Barrett \& Burdett (1981). The parabola fitted by their 
procedure would not have its axis identical with that of the cell and therefore also would give rise (as did the parametric fit) to a discontinuity at the tip of the pole. Pointed poles in B. subtilis have never been observed in the electron microscope. Furthermore, as distinct from the experimental curves of Fig. 5, this parabola has its major curvature near the end of the pole instead of near the sides.

Elliptical- and Cassinian-shaped poles are shown in curves $\mathrm{C}$ and $\mathrm{D}$. Those forms were proposed by Oldmixon (1974) to account for the poles of bacteria generally. The pole shapes are calculated from parameters fitted by Oldmixon (1974) to measurements of one photograph of $B$. subtilis published by Santo et al. (1972). He reported that the Cassinian gave a better fit in the sense that the sums of the squared deviations were smaller for the latter than the former.

Curve E, shown in Fig. 6, was fitted to the zonal dome model:

$$
z=z_{\max }+(2 T / P) \ln \left[1-(\operatorname{Pr} / 2 T)^{2}\right]
$$

where $z$ are $r$ are the axial distance and radius of any point on the pole. This curve is completely specified by the value of $a$ and $z_{\max }$ since $P / T$ can be calculated from equation (1) (the curve shown was fitted to the data from the present paper with the same $a$ and $z_{\max }$ values as for curve B). $P / T$ as evaluated from equation (1) is $3 \cdot 72$.

The parameter $P / T$ can also be estimated quite simply from the slope of a pole generated by a narrow growth zone. This can be done directly from equation (1) of Koch et al. (1981a):

$$
P / T=\left(2 \sqrt{1+S^{2}}+\delta S\right) / r ; S=\tan (\alpha)
$$

Thus, $P / T$ can be calculated from the angle $\alpha$ (taken as negative for constrictive growth), the radius of the pole at the same point, and from $\delta$, the thickness of the septum. The zonal dome model is the more specific model in that $\delta$ is set equal to 2 . For this second approach $P / T$ equals 3.82 , which yields a curve not much different than curve $E$ (curve not shown).

The first three curves of Fig. 6 are simply empirical approximations; the last two have possible theoretical bases. Curve D, the Cassinian, has the shape (see Oldmixon, 1974) that gives the cap of a pressure vessel with cylindrical sides the greatest ability to withstand internal pressure. Curve $\mathrm{E}$, the zonal dome, is the result of a specific wall growth mechanism. As applied to the Gram-positive rod, the ellipse and the Cassinian show small discontinuities at the cylinder-pole border - possibly too little when compared with electron photomicrographs. On the other hand, the polynomial, axial parabola and the zonal dome have too great a discontinuity. We conclude that none of the curves shown in Fig. 6 is a reasonable approximation of the pole shape of $B$. subtilis such as shown in Fig. 3.

\section{Comparison of the diameter of curvature with the narrow growth zone model}

Although $P / T$ is a little different when estimated by the two approaches described in the last section, Table 3 shows the diameter of curvatures for the peripheral $45^{\circ}$ of arc and the central $90^{\circ}$ of arc averaged for the three cell populations and compared with the predictions of equation (3) for either of the two ways of determining $P / T$ based on equations (1) and (7). Based on either approach, the zonal dome model also fares poorly.

\section{Table 3. Diameters of curvature of Bacillus subtilis}

\begin{tabular}{lccc} 
Central $90^{\circ}$ arc & $\begin{array}{c}\text { Average diameter } \\
\text { observed }(\mu \mathrm{m})^{*}\end{array}$ & $\begin{array}{c}\text { Diameter predicted by the } \\
\text { zonal dome model }(\mu \mathrm{m})\end{array}$ \\
Peripheral $45^{\circ}$ arc & 0.598 & 1.393 & 1.302 \\
$P / T=3.72 \ddagger$ \\
\hline
\end{tabular}

* Average of measurements on completed poles of 79 cells.

$\dagger P / T$ fitted from mean $\alpha$ and $a$ measurements from equation (7) as applied to the base of the poles for the zonal dome model $(\delta=2)$

$\ddagger P / T$ fitted from mean $z_{\max }$ and $a$ measurements from equation (1). 
Fit of completed poles to full narrow growth zone model with variable $\delta$

None of the models given above appear to give a suitable description of the shape of the pole of $B$. subtilis. A priori, the zonal dome theory of Koch et al. $(1981 a, b)$ has the most reasonable biological basis, but it deviates, as we have seen, in that actual cells have initial diameters of curvature smaller than predicted and have final curvatures larger than predicted. Moreover, the discontinuity with the cylindrical side wall of actual cells is less than that predicted.

Instead of rejecting the narrow growth zone model in its entirety, we can modify it and assume that the thickness of the splitting septum, $\delta$, is not a constant throughout the pole formation process. In one of the earlier papers (Koch et al., 1982), this approach was used to provide an explanation for the spherical shape of staphylococci. This was done by assuming that $\delta$ is initially very small and increases during pole development toward 2 . For the present case, a good fit can be achieved by assuming that initially $\delta$ is greater than 2 and then decreases. This is consistent with electron microscopic evidence, such as that illustrated by Fig. 1 of Burdett \& Higgins (1978), which shows that the septum at the stage where it first splits is considerably thicker than twice the wall thickness of the external stress-bearing wall. However, the micrographs show that the wall thickness at the point of splitting becomes a little thinner than twice the external wall thickness, but then thickness increases again to become about twice as thick as the external wall. It is a mathematical requirement of the narrow growth zone model that $\delta$ becomes greater than 2 for splitting and separation of daughter cells to occur. These considerations suggest that the quantity $\delta$ be taken as an observed variable instead of assuming that it is constant at a value of 2 as assumed by the simple zonal dome theory. Figure 7 shows the calculated pole shape for various regima for decrease in $\delta$ to a final value of 2 . In this fitting we assumed that $\mathrm{P}_{a} / T$ is constant and equal to 1 instead of a value of about 1.2 to $1 \cdot 3$. This is the range that best fits the zonal dome theory. (Curve $E$ of Fig. 6 was calculated for $\mathrm{P}_{a} / T=1 \cdot 22$.) The value of 1 is an especially reasonable choice because, as discussed elsewhere (Koch et al., $1981 b, 1982$; Koch, 1983), this is the value of $\mathrm{P}_{a} / T$ needed for the side walls of these organisms which elongated by the entirely distinct process of inside-to-outside growth. In this process, the diffuse addition of murein units occurs outside the cytoplasmic membrane and where linkage to form a loose network occurs. Later, these units become extended as the layer moves outward and comes to bear the tension created by the hydrostatic pressure within the cell. It can be seen that a narrow growth zone model, assuming variation in $\delta$ consistent with observation, gives the experimentally observed shapes, i.e. initially a small diameter of curvature and centrally less curved than the diameter of the original cylinder.

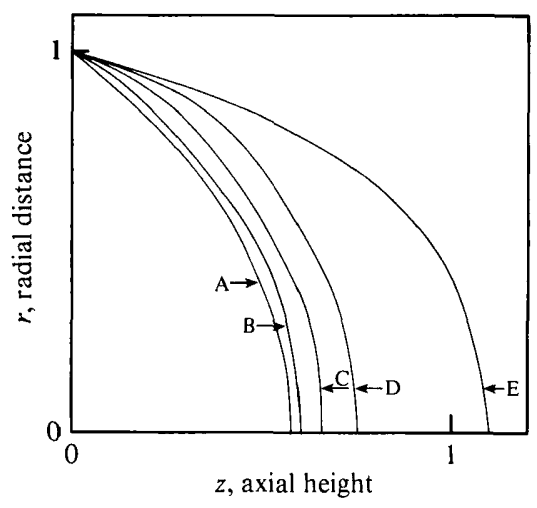

Fig. 7. The shape of cell poles predicted by a narrow growth zone with variable $\delta$. Curve $\mathrm{A}, \delta=2$ (the zonal dome model); Curve B, $\delta$ initially 3 , decreasing linearly to 2 when $z=0 \cdot 1$; Curve C, $\delta$ initially 4 , decreasing to 2 when $z=0 \cdot 2$; Curve $\mathrm{D}, \delta$ initially 4 , decreasing to 2 when $z=0 \cdot 4$; Curve $\mathrm{E}, \delta$ initially 4 , decreasing to 2 when $z=1 \cdot 0$. In all cases, once $z$ became 2 it remained at this value for the rest of the developmental process. 


\section{Split-and-stretch model}

The narrow growth zone model, even with $\delta$ taken as a variable, does not account for the differences in angles and diameter of curvatures between the nascent poles and the completed poles of the same cell. At the least, we must modify the narrow growth zone model by permitting some continued expansion after the septal wall is externalized. Alternatively, we propose a new model, the 'split-and-stretch' model based on previous ideas of Higgins \& Shockman (1976) and Burdett \& Higgins (1978). This model assumes that the septum is created under polymerization conditions that lead to no stress until the septum starts to split. In its most general form the full surface stress forces at some rate the split portion into its maximally extended conformation, increasing its original area. But the splitting of the external wall also places stress on the unsplit regions of the septum. Because the region is thicker and because the components of stress are smaller, this wall stretches more slowly and to a lesser degree. Although a limited amount of new murein is added to largely completed septa and even to separated poles (Koch, 1983), this presumably occurs immediately adjacent to the cytoplasmic membrane. In those cases where closing the annulus is not too close to the splitting septum, the murein added to the inner surface of the annulus probably will not be stretched before further new material is added to it.

In this paper, calculations are presented of pole shapes if the externalized wall doubles its area and the unsplit cross-wall stretches to a variable degree as pole formation takes place. To calculate the expected pole shape for a given pattern of expansion, the final area to which the unsplit septum of radius $r$ is capable of expanding is equated to the sum of the new increment of fully expanded area of newly externalized wall and the potential fully expanded area of the remaining septum. We define $E$ as the ratio of fully expanded area of the completely unsplit septum of radius $a$ to its actual area and give it a constant value of 2, and $F$ as the ratio of the area to which it will eventually expand to the actual area of unsplit septum. Note that $F$ is a factor that goes from 1 to $E$ as the radius of the unsplit septum $r$ decreases from $a$ to zero. The area yet to be added to the evolving pole is $\pi r^{2} E / F$ where an infinitesimal annular split takes place, the area of fully expanded external wall so created, is $2 \pi r \sqrt{1+S^{2}} \mathrm{~d} z$ and the area that remaining septal wall would have if fully expanded is $\pi(r+\mathrm{d} r)^{2} E / F$. On equating the first quantity to the sum of the other two, expanding the binomial, dividing by $2 \pi r$, and dropping the second-order term, we obtain:

$$
E \mathrm{~d} r / F=\sqrt{1+S^{2}} \mathrm{~d} z
$$

Since $\mathrm{d} r / \mathrm{d} z$ is by definition $S$, this equation can be solved to yield:

$$
S=\left(E^{2} / F^{2}-1\right)^{-1 / 2}
$$

Consequently, the shape of a pole can be calculated from any assumed path for $F$ to change from 1 to $E$ as the septum splits.

This equation applies when $F$ changes progressively with the splitting of the septum. If it changes instantaneously to $E$, the septum can remain planar and the split material lead to cylindrical extension. In the extreme case (see Koch, 1983) of facile expansion, the final pole consists of a cylindrical extension whose length is half the radius and planar end. On the other hand, if the unsplit septum $(F=1)$ does not stretch at all, then a conical pole results (see Koch, 1983). These shapes are shown with dashes in Fig. 8. The intermediate shaped poles are not too unrealistic, particularly the curve marked 1.4 , and could be matched much more closely by the assumption of a non-linear change in $F$. Each curve is marked with a number that denotes the linear rate that $F$ increases from 1 to the maximum expansion of two-fold assumed for this illustrative example.

The curves calculated in this way should correspond to the nascent pole of a newly divided cell. But of course this pole shape does not enclose the maximum volume. Consequently, with relatively few cuts and/or shifting of strands, it is not surprising that a more hemispherical shape, such as is actually present in the completed pole, would be later achieved.

A quite similar model was considered by Higgins \& Shockman (1976); they found it unapplicable to $S$. faecalis in that the wall does not appear to expand very much. This model was 


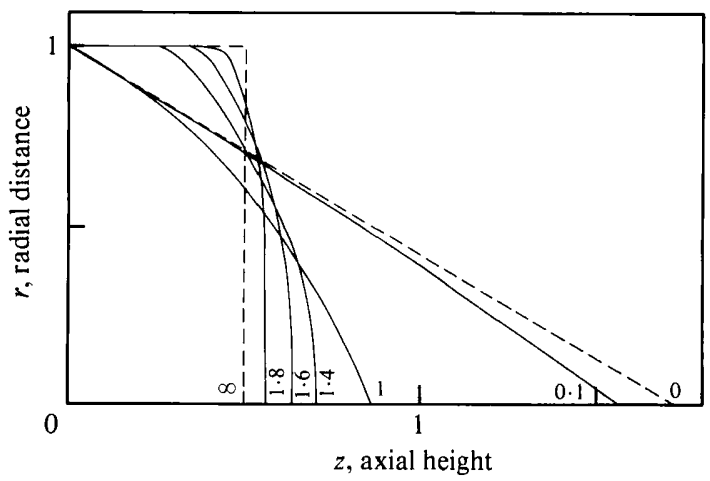

Fig. 8. The shape of cell poles predicted by the 'split-and-stretch' model. Profiles of poles predicted by the model for various laws for change in $F . F$ is the factor of area expansion which is initially 1 and linearly increases to an upper limit of $F=E$. $E$ is the maximum factor of expansion exhibited by the externalized and split wall and was taken to be 2 for these calculations. The rates of increase of $F$ per unit axial distance are indicated on the curve. For the smallest value, $F$ remains at 1 ; for the largest, $F$ was 2 throughout the splitting process. Note that a spherical pole is the thermodynamically most stable shape and all of these poles would, subsequent to formation, tend to become more spherical.

also considered for $B$. subtilis by Burdett \& Higgins (1978); they calculated the expansion factor that the newly externalized septal wall had to undergo. Their expansion factor varied from 4.5 at the start of splitting of the cross wall to 1.3 at the end of cell division. This would be consistent with a good deal of expansion of the unsplit septal wall when surface stress was initially applied; i.e. $F$ rises from 1 to $E$ rapidly during the splitting process.

The externalization due to septal splitting probably results from the same autolytic enzymes that promote inside-to-outside growth of the cylindrical wall by hydrolysis of outermost layers (see Rogers et al., 1980). Whilst their action may be more concentrated in the septal region (Burdett, 1980a), even if they were largely or solely secreted on the cylindrical wall they would quickly reassociate on murein elsewhere and thus may indirectly cause some hydrolysis of pole wall both for splitting and for limited surface hydrolysis. The evidence of this movement is that chain-forming mutants deficient in lytic enzymes grow and separate if co-cultured with wildtype bacilli (Fein \& Rogers, 1976; Rogers et al., 1974; Pooley, 1976) instead of growing as filaments. Additional evidence is that the addition of proteases to growing cultures leads to chain formation in cells with wild-type amidase (Jolliffe et al., 1981).

Finally, we note that there is a totally different model that also generates a pole that is initially flattened and has a smaller curvature near the edge than on the axis of the pole and does change progressively after completion. This is the variable $T$ model (Koch, 1982, 1983), based on the assumption that the wall growth process is one of intercalation. While this process can occur all over the surface of the cell, its qualitative nature is altered in the central region of the cell that is destined to become the site of cell division. The change is one that decreases the value of $T$ several-fold. Even though a high positive pressure is present inside the bacteria, further growth leads to invagination because of the biophysical consequences of the change in $T$. Such a constrictive process leads to an initially flat-ended cell. However, as $T$ returns to the value characteristic for growth of the side walls, the pole grows more hemispherical and eventually becomes just a little bit more pointed than a hemisphere. While these changes are qualitatively the same as seen here [and as we have found in B. megaterium (I. D. J. Burdett \& A. L. Koch, unpublished observations)], the mechanism is only logically applicable for Gram-negative cases. With Bacillus a thick septum is formed as an addition to the inside of the stress-bearing cylindrical wall and which later splits. On the other hand, for the Gram-negative rod there is an invagination (a constriction) that does not appear to change the topology of the sacculus; i.e. it is a simple infolding and not the formation of an annulus that is simply amended to the external wall. Both the septal formation and constriction processes are related in that they both provide a way to translate biochemical work into the work needed to divide the cell into daughters. 
This work was started during a tenure of a Guggenheim Fellowship to A.L.K. It was completed with the aid of a travel grant from the Burroughs Wellcome Company. We thank Jane Whalley, Jeff Smith, John Green and Jeff Collins for assistance in the analysis of electron micrographs. The junior author thanks the senior author and $\mathrm{Dr}$ Howard J. Rogers for their hospitality.

\section{REFERENCES}

Barrett, A. N. \& BurdetT, I. D. J. (1981). A threedimensional model reconstruction of pole assembly in Bacillus subtilis. Journal of Theoretical Biology 92, 127-139.

BURDETT, I. D. J. (1980a). Analysis of sites of autolysis in Bacillus subtilis by electron microscopy. Journal of General Microbiology 120, 35-49.

BURDETT, I. D. J. (1980 b). Quantitative studies of rodcoccus morphogenesis in a temperature-sensitive rod-mutant of Bacillus subtilis. Journal of General Microbiology 121, 93-103.

BurdetT, I. D. J. \& Higgins, M. L. (1978). Studies of pole assembly in Bacillus subtilis by computer reconstruction of septal growth zones seen in central, longitudinal, thin sections of cells. Journal of Bacteriology 133, 959-971.

FeIN, J. E. \& Rogers, H. J. (1976). Autolytic enzymedeficient mutants of Bacillus subtilis 168. Journal of Bacteriology 127, 1427-1442.

Higgins, M. L. (1976). Three-dimensional reconstruction of whole cells of Streptococcus faecalis from thin sections of cells. Journal of Bacteriology 127, 13371365.

Higgins, M. L. \& Shockman, G. D. (1976). Study of a cycle of cell wall assembly in Streptococcus faecalis by three-dimensional reconstructions of thin sections of cells. Journal of Bacteriology 127, 1346-1358.

Jolliffe, L. K., DoYle, R. J. \& Streips, U. N. (1981). The energized membrane and cellular autolysis in Bacillus subtilis. Cell 25, 753-763.

KoCH, A. L. (1982). On growth and form of Escherichia coli. Journal of General Microbiology 128, 2527-2540.
KoсH, A. L. (1983). The surface stress theory of microbial shapes. Advances in Microbial Physiology 24, 301-366.

Koch, A. L., Higgins, M. L. \& Doyle, R. J. (1981 $a$ ), Surface tension-like forces determine bacterial shapes: Streptococcus faecium. Journal of General Microbiology 123, 151-161.

Koch, A. L., Mobley, H. L. T., Doyle, R. J. \& STREIPS, U. N. (1981 $b$ ). The coupling of wall growth and chromosome replication in Gram-positive rods. FEMS Microbiology Letters 12, 201-208.

Koch, A. L., Higgins, M. L. \& Doyle, R. J. (1982). The role of surface stress in the morphology of microbes. Journal of General Microbiology 128, 927945.

Oldmixon, E. (1974). The shapes of bacteria. PhD dissertation, Temple University School of Medicine, Philadelphia, Pa, USA.

PoOLeY, H. M. (1976). Turnover and spreading of old wall during surface growth of Bacillus subtilis. Journal of Bacteriology 125, 1127-1138.

Rogers, H. J., Pooley, H. M., Thurman, P. F. \& TAYLOR, C. (1974). Wall and membrane growth in bacilli and their mutants. Annales de microbiologie 125b, 135-147.

Rogers, H. J., Perkins, H. R. \& Ward, J. B. (1980). Microbial Walls and Membranes. London: Chapman and Hall.

Santo, L., Leighton, T. J. \& Doi, R. H. (1972). Ultrastructural analysis of sporulation in a conditional serine protease mutant of Bacillus subtilis. Journal of Bacteriology 111, 248-253. 